\title{
Cues from Sri Lankan Traditional Medicine to the Modern Drug Development Pipeline - for a Sustainable Future
}

\author{
Vindya Udalamaththa ${ }^{1}$, Udaya Samaratunga ${ }^{2}$ \& Preethi Udagama ${ }^{1 *}$ \\ 1. Department of Zoology and Environment Sciences, Faculty of Science, University of Colombo \\ 2. Department of Ayurveda Basic Principles, Faculty of Indigenous Medicine, \\ Gampaha Wickramarachchi University of Indigenous Medicine \\ ${ }^{*}$ Corresponding Author
}

ABSTRACT
Worldwide, traditional medicine (TM) systems based on cultural and
religious beliefs, and geographical regions of origin, possess their own unique
practices. The use of herbal preparations in TM follows the basic principle of
treating a disease by stabilizing organ systems of the body, with simultaneous
targeting of multiple symptoms. Time-tested practices from TM preparations,
fed into the development of modern drugs, can drastically reduce its long,
arduous turnover time. However, novel drug development research focusses
on single compound drugs; yet frequently, it is the synergistic activity of
phytocompounds in the original herbal preparation that renders them unique
in their therapeutic activity. A recent area of research examines the synergistic
activity of several compounds achieving multi drug targets, as a powerful
treatment regimen for diseases with complex aetiology.
Blessed with a lavish biodiversity and a rich, unique TM heritage, Sri
Lanka is well-placed to identify and produce drugs for a wide range of disorders.
Local research on diverse disorders has demonstrated promising results, yet
the standardization of effective drugs and supplements which compete with
the international market poses a significant challenge. State support as well as
the sustainable use of traditional medicinal plants are required. The cultivation
of medicinal plants would reduce their harvest in the wild, and help preserve
these species from extinction. Thus, a market of high value could be developed
for medicinal plant cultivators and the herbal pharmaceutical industry, which
would sanction its socio-economic growth and sustainable future.

KEYWORDS:
Sri Lanka, Traditional Medicine, polyherbal preparations, modern
drug development, synergism, international pharmaceutical market,
translational research

Suggested Citation: Udalamaththa, V., Samaratunga, U. and Udagama, P. (2021). Cues from Sri Lankan Traditional Medicine to the Modern Drug Development Pipeline - for a Sustainable Future. University of Colombo Review (New Series III), 2(2), 88 - 106.

(C) 2021 The Authors. This work is licenced under a Creative Commons Attribution 4.0 International Licence which permits unrestricted use, distribution, and reproduction in any medium, provided the original work is properly cited. 


\section{Introduction}

The World Health Organization (WHO) has defined Traditional Medicine (TM) as "the sum total of the knowledge, skills and practices based on the theories, beliefs and experiences indigenous to different cultures, whether explicable or not, used in the maintenance of health, as well as in the prevention, diagnosis, improvement or treatment of physical and mental illnesses" (WHO, 2000). In some countries TM is also termed alternative, complementary or non-conventional medicine. TM systems in different countries vary drastically, depending on religious beliefs, culture, history, and biodiversity from which medicinal material is extracted (WHO, 2000). However, in every TM system, the use of plants to prepare herbal medicines remains an important practice. The WHO defines traditional herbal medicines as "naturally occurring, plant-derived substances with minimal or no industrial processing that have been used to treat illness within local or regional healing practices (Tilburt \& Kaptchuk, 2008). World renowned TM systems such as Ayurveda in India, or Chinese, African, South American and Arabic traditional medicine systems use herbal medicine for the primary healthcare needs of $75 \%$ of the world's population (Pan et al., 2014). In countries such as India and Sri Lanka, archaeological proof of using herbal medicine to treat a wide range of disorders has a history of more than 5000 years (Edwards et al., 2012). There is also written evidence such as Arkaprakasa (pharmacology and pharmacy) and Kumaratantra (paediatric diseases and management) claimed to be documented by King Ravana of Sri Lanka, which are now considered basic reference pharmacopoeias in Ayurveda (Sharma, 2000).

Traditional herbal preparations, although having a long record of use and acceptance by patients, are not within mainstream medical treatment. This is due to the absence of scientific proof and standardization according to international drug manufacturing protocols. However, the WHO general guidelines for Methodologies on Research and Evaluation of Traditional Medicine (WHO, 2000) supports proceeding directly to the clinical evaluation of suitable time-tested medicinal preparations, without carrying out preclinical and toxicological studies. Traditional health practitioners, however, consider it their responsibility to continue with their treatment of patients in need, and generally ignore requests to commercialize their knowledge of herbal preparations handed down from generation to generation.

Modern drug discovery and development has reached an immensely influential level, with the use of cutting edge, high throughput screening devices and computational drug target predictions. Although, an estimated USD 2.6 billion is spent to develop a new treatment, most potential drug candidates are rejected between Phase I trials and regulatory approval (Fleming, 2018). Leading biopharmaceutical companies such as Pfizer, Roche, and Sanofi are already using artificial intelligence (AI) driven systems to invent new drugs which are cheaper and more effective with less turnaround time. Yet, there are no approved drugs produced to date with the help of AI systems, as its use in drug discovery is contentious (Fleming, 2018).

Most vital drugs approved in recent decades are reported to be isolated from plants. The naturally occurring, bioactive compounds are termed "phytochemicals". They are 
plant secondary metabolites, which are not required for the immediate survival of the plant, but which can be synthesized to increase its robustness for better survival (Kennedy \& Whiteman, 2011). With the first breakthrough in the 18th century when morphine was isolated from the plant Papaver somniferum, numerous effective drugs such as salicylic acid (the precursor of aspirin), cocaine, quinine, and digitoxin have been discovered this way (Pan et al., 2009).

The modern drug pipeline relies, however, on single compounds as potential drugs. It does not consider the value of multi- herbal mixtures or whole extracts of a single herbal plant. Traditional medicine preparations, on the other hand, focus on the synergy of a myriad of "phytochemicals". Even though cues extracted from prominent traditional medicine systems are tested globally, the search for a single compound drug may compromise their effectiveness as suitable treatments for diseases in clinical trials. In TM, poly herbal preparations are considered to exert synergistic interactions increasing bioavailability of the compounds, thereby enhancing the therapeutic effects and reducing toxicity (Jia et al., 2004). In the context of TM, it is believed that a herbal preparation maintains or stabilizes different systems of the body, explaining these multitarget effects by a sequence of compounds in a preparation. It is now apparent that whole extracts or polyherbal mixtures could be effective due to the synergistic activity of the different phytocompounds present, as important bioactivities tend to lose their effectiveness upon fractionation (Caesar \& Cech, 2019). However, the phenomenon of antagonistic effects has also been studied, as this could mask important bioactivities in a polyherbal mixture or a whole extract.

\section{The Role of Synergism}

In recent years, the study of the synergistic effects exerted by whole plant preparations has been considered extremely important in pharmaceutical pipelines, and advanced techniques in metabolomics and biochemometrics are constantly used to identify underlying mechanisms of actions alongside toxicity and the side effects of such preparations (Caesar \& Cech, 2019).

A review of synergistic bioactivities in Chinese TM (CTM) multi herbal preparations on diseases such as cancer, asthma, myocardial ischemia, cardiovascular diseases, hepatic injury and fungal disorders, reported confirmation of synergistic activity in the compounds present in the preparations (Zhou et al., 2016). The review also reported an increased use of proper mathematical or computational methods in detecting synergistic activity of CTM preparations between the years 2000 to 2014 (Zhou et al., 2016). In a study on South African $\mathrm{TM}$, effective anti-gonococcal activity was reported in the use of different combinations of 7 medicinal plants (Vambe et al., 2018). In India, where Ayurvedic literature such as the Sarangadhar Samhita concentrated on synergistic activity of ingredients ("Samyoga"), there is now a move to focus on a network of pharmacology research to investigate herbal preparations exerting synergistic activity (Mukherjee et al., 2018). In this context, Lagenaria siceraria, a food plant used in Indian TM against hyperlipidaemia, and in Sri Lankan TM to treat lactation issues, fractures, and inflammation, was studied and multiple compounds in 
the extract targeting multiple sites of relevant pathways building up synergistic interactions were reported (Mukherjee et al., 2018).

\section{Sri Lankan Traditional Medicine}

Sri Lanka has a unique system of traditional medicine, supported by archaeological evidence of a complete hospital system and medical instruments similar to those currently in use (Kannangara, 2016). Sri Lankan TM is divided into different traditions, based on geographical regions and practices. It is often mistaken as a descendent of the Ayurveda of India, but there are clear cut differences between Indian and Sri Lankan TM systems. For example, preparation methods and the use of Arka (distilled products of herbal preparations) is known to have been introduced to Indian Ayurveda at a later stage - approximately in the 12th Century, whereas the word Arka-prakasha is first cited in ancient dialogues of a Sri Lankan story, linking its roots to Sri Lanka (Jalpa \& Pandya, 2017). Sri Lankan TM practitioners often use medicinal plants endemic to Sri Lanka, in their herbal preparations. They also replace the rare herbs used in Indian Ayurveda with easily accessible plants in Sri Lanka. For example, instead of Shalaparni (Desmodium gangeticum) and Daruharidra (Berberis aristate) used in Indian herbal preparations, they use Aswenna (Alysicarpus vaginalis) and Weniwelgata (Coscinium fenestratum) respectively.

Although there is a large number of TM practitioners throughout the county specializing in different disorders such as diabetes, arthritis, fractures, injuries, different types of cancers and eye disorders, it is rarely that patient feedback is gathered and analyzed. Further, neither the preparation methods nor the herbs used in the treatments are disclosed to the general public by most Sri Lankan TM practitioners. Therefore, the potential to scientifically validate and commercialize these products is severely restricted. However, as the herbal preparations have been used for a long time, and TM practices in the country are valued, practitioners do not view scientific validation and commercialization as an obligatory step forward. For the academics amongst TM and Ayurvedic practitioners, however, documenting the effects of herbal preparations in usage, and disseminating this knowledge to the scientific community for the greater good of humanity, is of great importance.

\section{Conservation and Sustenance of Medicinal Plants in Sri Lanka}

Countries such as India and China which are the leading exporters of herbal products, focus on sustainable agricultural development to meet the increasing global demand for their standardized, safe and efficient products. They focus on socio-economical sustainability through the organic farming of herbs resulting in high market values for the raw material required for quality products, thereby safeguarding economic growth and social stability (Shahrajabian et al., 2019; Kumar et al., 2021). If the Sri Lankan indigenous herbal preparation and drug development process is streamlined along the aforementioned lines, the achievement of a sustainable future through the flourishing cultivation of medicinal plants can be achieved. 
In 2004, the World Bank's Indigenous Knowledge Notes described the methods used in Sri Lanka in the conservation and sustainable use of medicinal plants - a project funded in 1998 with a US \$4.6 million grant by the Global Environment Facility. The project had successfully implemented a) in situ conservation, mainly by establishing 5 medicinal plant conservation areas in Bibile, Ritigala, Rajawake, Naula, and Kanneliya; b) ex situ conservation by promoting home-gardens and nursery cultivation; and c) providing institutional and informational support to the area on medicinal plants (Mahindapala, 2004). According to a survey carried out in 2002, Sri Lanka has about 1432 medicinal plant species, of which 148 are endemic. The number of threatened species is estimated at 110 , prompting action on the sustainable use of medicinal plants (Ratnayake \& Kariyawasam, 2007). Several large-scale medicinal plant gardens have been established and at present, maintained by different authorities such as the Ministry of Agriculture, the Ministry of tourism, and the Ministry of health, nutrition and indigenous medicine. The Ganewatte medicinal plant garden is one such garden in the intermediate zone of the country, occupying 22 acres of land, with medicinal plants that help in conservation, economic development and educational programmes (Ganewatte Medicinal Plant Gardens, 2009). In addition, private non-profit organizations such as the Nagenahiru Foundation have established medicinal plant arboretums and herbariums to propagate rare plants, educate, demonstrate, and conduct field research promoting the sustainable use of threatened and rare medicinal plants under the guidance of the National Institute of Traditional Medicine (Nagenahiru Foundation, 2010). A renowned cosmetic herbal product manufacturer, Nature's Beauty Creations, has pledged 100,000 medicinal plants and 5 million medicinal plant seeds by 2025 to the government, as a part of its environment protection campaign sadaharitha hetak. The company also possesses the largest privately owned medicinal plant collection, grown and maintained by its agriculture department (Daily FT, 2021).

In the following section, publications on Sri Lanka's TM herbal preparations that have effectively targeted diseases in vitro, and during patient treatment are listed. Under each subsection, a summary of research carried out is presented, together with a list of medicinal plants used (either as whole extracts of plant parts or as polyherbal mixtures).

\section{Research Based on Cues from Sri Lankan Traditional Medicine}

Tables 1 to 5 present studies on anti-cancer, hypoglycaemic, hypolipidaemic and anti-obesity and anti-inflammatory effects of Sri Lankan TM preparations.

Scientifically validated herbal preparations against oral squamous cell carcinoma (OSCC), laryngeal cancer cells, breast cancer, non-small cell lung cancer, human hepatoma, endometrial carcinoma, cervical adenocarcinoma, and rabdomyosarcoma are listed in table 1 with stage of study and findings. 
Table 1

Cancer Chemopreventive Activity of Sri Lankan Herbal Preparations

\begin{tabular}{|c|c|c|c|}
\hline Herbal preparation & Cancer type & $\begin{array}{l}\text { Stage of } \\
\text { study }\end{array}$ & Findings \\
\hline $\begin{array}{l}\text { 1. A mixture of powdered } \\
\text { fruits of Emblica } \\
\text { officinalis (Indian } \\
\text { gooseberry) and } \\
\text { Tinospora cordifolia } \\
\text { (Heart-leaved } \\
\text { moonseed) }\end{array}$ & $\begin{array}{l}\text { OSCC of the buccal } \\
\text { mucosa and the } \\
\text { tongue }\end{array}$ & $\begin{array}{l}\text { Clinical } \\
\text { trial }\end{array}$ & $\begin{array}{l}\text { 1) OSCC buccal mucosa } \\
\text { with greatly reduced } \\
\text { local and overall } \\
\text { recurrences } \\
\text { 2) OSCC of the tongue, } \\
\text { showed statistically } \\
\text { significant reduction } \\
\text { in nodal and overall } \\
\text { recurrences (Dias et al., } \\
\text { 2018) }\end{array}$ \\
\hline $\begin{array}{l}\text { 2. Decoction of } \\
\text { Adenanthera pavonina } \\
\text { L. (red sandalwood) } \\
\text { and Thespesia } \\
\text { populnea L. (Pacific } \\
\text { rosewood) }\end{array}$ & $\begin{array}{l}\text { Laryngeal cancer } \\
\text { cells } \\
\text { (Hep- } 2 \text { cell line) }\end{array}$ & In vitro & $\begin{array}{l}\text { Anti-proliferative effects } \\
\text { and apoptosis of cells. } \\
\text { (Lindamulage \& Soysa, } \\
\text { 2016) }\end{array}$ \\
\hline $\begin{array}{l}\text { 3. Decoction of Flueggea } \\
\text { leucopyrus (Wild) } \\
\text { (Purple tephrosia) }\end{array}$ & $\begin{array}{l}\text { Breast cancer } \\
\text { (MCF-7, } \\
\text { MDA-MB-231 and } \\
\text { SKBR-3 cell lines) }\end{array}$ & In vitro & $\begin{array}{l}\text { Cytotoxic effects by } \\
\text { inducing apoptosis. } \\
\text { (Mendis et al., 2015) }\end{array}$ \\
\hline $\begin{array}{l}\text { 4. A polyherbal mixture } \\
\text { containing Nigella } \\
\text { sativa (Black cumin) } \\
\text { (seeds), Hemidesmus } \\
\text { indicus (Indian } \\
\text { Sarsaparilla) (roots) } \\
\text { and Smilax glabra } \\
\text { (Chinaroot) (rhizomes) }\end{array}$ & $\begin{array}{l}\text { Non-small cell lung } \\
\text { cancer (NCI-H292 } \\
\text { cells) }\end{array}$ & In vitro & $\begin{array}{l}\text { Cytotoxic effects by } \\
\text { inducing apoptosis, anti- } \\
\text { oxidant activity and colony } \\
\text { formation inhibition. } \\
\text { (Pathiranage et al., 2020) }\end{array}$ \\
\hline $\begin{array}{l}\text { 5. Aqueous and ethanolic } \\
\text { extracts of a polyherbal } \\
\text { mixture comprised of } \\
\text { Nigella sativa (seeds), } \\
\text { Hemidesmus indicus } \\
\text { (roots) and Smilax } \\
\text { glabra (rhizome) }\end{array}$ & $\begin{array}{l}\text { Human hepatoma } \\
\text { (HepG2 cell line) }\end{array}$ & In vitro & $\begin{array}{l}\text { Cytotoxicity (Samarakoon } \\
\text { et al., 2010) }\end{array}$ \\
\hline
\end{tabular}




\begin{tabular}{|c|c|c|c|}
\hline Herbal preparation & Cancer type & $\begin{array}{l}\text { Stage of } \\
\text { study }\end{array}$ & Findings \\
\hline $\begin{array}{l}\text { 6. Non-polar extracts of } \\
\text { Garcinia. zeylanica } \\
\text { (Ela goraka) and } \\
\text { Garcina quaesita } \\
\text { (Indian tamarind), } \\
\text { S. castaneifolia barks }\end{array}$ & $\begin{array}{l}\text { Breast cancer } \\
\text { (MDA-MB-231 and } \\
\text { MCF-7) }\end{array}$ & In vitro & $\begin{array}{l}\text { Inhibition of proliferation } \\
\text { of breast cancer stem cells } \\
\text { (Rajagopalan et al., 2018) }\end{array}$ \\
\hline $\begin{array}{l}\text { 7. Decoction of the Aerial } \\
\text { Parts of Flueggea } \\
\text { leucopyrus (Purple } \\
\text { tephrosia) }\end{array}$ & $\begin{array}{l}\text { Endometrial } \\
\text { Carcinoma (AN3CA } \\
\text { cells) }\end{array}$ & In vitro & $\begin{array}{l}\text { Cytotoxicity via apoptosis. } \\
\text { (Samarakoon et al., 2014) }\end{array}$ \\
\hline $\begin{array}{l}\text { 8. Polyherbal medicine } \\
\text { "Le pana guliya" }\end{array}$ & $\begin{array}{l}\text { Hepatocellular } \\
\text { carcinoma and } \\
\text { human cervical } \\
\text { adenocarcinoma } \\
\text { (HepG2 and HeLa } \\
\text { cells) }\end{array}$ & In vitro & $\begin{array}{l}\text { Cytotoxicity via oxidative } \\
\text { stress dependant apoptosis. } \\
\text { (Wageesha et al., 2017a) }\end{array}$ \\
\hline $\begin{array}{l}\text { 9. Polyherbal medicine } \\
\text { "Le pana guliya" }\end{array}$ & $\begin{array}{l}\text { Human } \\
\text { Rhabdomyosarcoma } \\
\text { cell line (RD), and } \\
\text { human breast } \\
\text { adenocarcinoma cell } \\
\text { line (MCF-7) }\end{array}$ & In vitro & $\begin{array}{l}\text { Strong cytotoxicity activity. } \\
\text { (Wageesha et al., 2017b) }\end{array}$ \\
\hline
\end{tabular}

Herbal preparations against hyperglycaemia including water and chemical extracts of single and herbal mixtures, powders, and drinks are listed in Table 2, with stage of study, herbal combinations used, and findings.

Table 2

Sri Lankan Herbal Preparations against Hyperglycaemia

\begin{tabular}{|l|l|l|}
\hline Herbal preparation & Study stage & Findings \\
\hline $\begin{array}{l}\text { 1. Ethanol and water extracts } \\
\text { of Munronia pinnata (Bin } \\
\text { kohomba) }\end{array}$ & $\begin{array}{l}\text { Pre-clinical study } \\
\text { using Wista rats }\end{array}$ & $\begin{array}{l}\text { Significant hypoglycaemic } \\
\text { effects by both extracts } \\
\text { (Hapuarachchi et al., 2011) }\end{array}$ \\
\hline $\begin{array}{l}\text { 2. Herbal powder derived from } \\
\text { 'Thalpathe Piliyam' }\end{array}$ & $\begin{array}{l}\text { Clinical study } \\
\text { using type II } \\
\text { diabetic patients }\end{array}$ & $\begin{array}{l}\text { Hypoglycaemic effects similar } \\
\text { to the allopathic treatment } \\
\text { regime. (Harshamali \& } \\
\text { Sakunthala, 2019) }\end{array}$ \\
\hline
\end{tabular}




\begin{tabular}{|c|c|c|}
\hline Herbal preparation & Study stage & Findings \\
\hline $\begin{array}{l}\text { 3. Hot water extract of } \\
\text { Tragia involucrate L. (Wel } \\
\text { Kahambiliya) }\end{array}$ & $\begin{array}{l}\text { Pre-clinical } \\
\text { study using } \\
\text { Streptozotocin } \\
\text { induced diabetic } \\
\text { rats }\end{array}$ & $\begin{array}{l}\text { Significant reduction in blood } \\
\text { glucose level, triglycerides, and } \\
\text { very low-density lipoprotein } \\
\text { cholesterol and increases high } \\
\text { density lipoprotein cholesterol } \\
\text { levels (Palli et al., 2017) }\end{array}$ \\
\hline $\begin{array}{l}\text { 4. Malewana Madhumeha } \\
\text { Choorna polyherbal hot } \\
\text { infusion }\end{array}$ & $\begin{array}{l}\text { Biochemical } \\
\text { assays }\end{array}$ & $\begin{array}{l}\text { Strong antioxidant, free radical } \\
\text { scavenging activity, } \alpha \text {-amylase } \\
\text { enzyme inhibition activity. } \\
\text { (Keshala et al., 2021) }\end{array}$ \\
\hline $\begin{array}{l}\text { 5. A polyherbal mixture } \\
\text { composed of leaves of } \\
\text { Murraya koenigii L. (Curry } \\
\text { leaves tree), cloves of Allium } \\
\text { sativum L.(Garlic), fruits } \\
\text { of Garcinia quaesita Pierre } \\
\text { (Rath goraka) and seeds of } \\
\text { Piper nigrum L. (Pepper) }\end{array}$ & $\begin{array}{l}\text { Pre-clinical } \\
\text { study using } \\
\text { streptozotocin } \\
\text { induced diabetic } \\
\text { rats }\end{array}$ & $\begin{array}{l}\text { Significantly lower glycated } \\
\text { haemoglobin, significantly } \\
\text { increased serum insulin } \\
\text { concentration, } \beta \text {-cell restoration } \\
\text { in the pancreas, restoration of } \\
\text { lipoproteins. } \\
\text { (Liyanagamage et al., 2021) }\end{array}$ \\
\hline $\begin{array}{l}\text { 5. Herbal drink of Syzygium } \\
\text { Cumini (Malabar plum) bark } \\
\text { extract }\end{array}$ & $\begin{array}{l}\text { Biochemical } \\
\text { assays }\end{array}$ & $\begin{array}{l}\text { Antiglycation and antioxidant } \\
\text { effects (Perera et al., 2014) }\end{array}$ \\
\hline $\begin{array}{l}\text { 6. Boiled aqueous extract of } \\
\text { Nyctanthes arbor-tristis L } \\
\text { (Night-flowering Jasmine) } \\
\text { flowers }\end{array}$ & $\begin{array}{l}\text { Pre-clinical study } \\
\text { using male mice }\end{array}$ & $\begin{array}{l}\text { Significant reduction of fasting } \\
\text { and random blood glucose } \\
\text { levels, decreased levels of total } \\
\text { cholesterol and tryglycerides, } \\
\text { and inhibition of glucose uptake } \\
\text { by the intestine. (Rangika et al., } \\
2015 \text { ) }\end{array}$ \\
\hline $\begin{array}{l}\text { 7. Methanolic extract of } \\
\text { Aporusa lindleyana (Kabella) } \\
\text { leaves }\end{array}$ & $\begin{array}{l}\text { Pre-clinical study } \\
\text { using Sprague- } \\
\text { Dawley rats }\end{array}$ & $\begin{array}{l}\text { Significant reduction of fasting } \\
\text { and random blood glucose } \\
\text { levels and no toxic signs in rats } \\
\text { (Samanmali et al., 2014) }\end{array}$ \\
\hline $\begin{array}{l}\text { 8. Aqueous leaf extract of } \\
\text { Passiflora suberosa (Corky } \\
\text { passion vine) }\end{array}$ & $\begin{array}{l}\text { Pre-clincal study } \\
\text { using male mice }\end{array}$ & $\begin{array}{l}\text { Reduction of fasting and } \\
\text { random blood glucose levels. } \\
\text { Tri glycerides, total cholesterol } \\
\text { and glucose uptake from the } \\
\text { intestine. (Sudasinghe \& Peiris, } \\
2018 \text { ) }\end{array}$ \\
\hline
\end{tabular}

Herbal preparations such as water and chemical extracts from herbs and "choorna" against hyperlipidaemia and obesity are listed in Table 3. 
Table 3

Sri Lankan Herbal Preparations against Hyperlipidaemia

\begin{tabular}{|c|c|c|}
\hline Herbal preparation & Study stage & Findings \\
\hline $\begin{array}{l}\text { 1. Cold water extract of new } \\
\text { herbal formulation consisting } \\
\text { of Allium sativum (Bulb) } \\
\text { (Garlic), Piper nigrum (Nuts) } \\
\text { (Pepper), Murrayakoenigii } \\
\text { (Curry leaf tree) (Leaves) }\end{array}$ & Clinical trial & $\begin{array}{l}\text { Significant decrease in } \\
\text { serum cholesterol levels; } \\
\text { triglycerides, LDL and } \\
\text { TC:LDL ration. (Pushpamali \& } \\
\text { Chandrasiri, 2016) }\end{array}$ \\
\hline $\begin{array}{l}\text { 2. Crude methanolic extract } \\
\text { of Emblica officinalis fruits } \\
\text { (Indian gooseberry) }\end{array}$ & $\begin{array}{l}\text { Preclinical study } \\
\text { using cholesterol } \\
\text { induced Wistar rats }\end{array}$ & $\begin{array}{l}\text { Significant decrease in total } \\
\text { cholesterol, triglycerides and } \\
\text { LDL and significant increase } \\
\text { in HDL (Dissanayake et al., } \\
\text { 2017) }\end{array}$ \\
\hline $\begin{array}{l}\text { 3. Traditional herbal "choorna" } \\
\text { consisting of Nigella sativa } \\
\text { (Black cumin), Saussurea } \\
\text { lappa (Indian costus root), } \\
\text { Zingiber officinale (Ginger), } \\
\text { Allium sativum (Garlic), } \\
\text { Cassia fistula (Pudding } \\
\text { pipe tree) and Cassia senna } \\
\text { (Indian Senna) }\end{array}$ & $\begin{array}{l}\text { Clinical trial using } \\
\text { overweight and } \\
\text { obese patients }\end{array}$ & $\begin{array}{l}\text { Significant decrease in the } \\
\text { BMI of patients } \\
\text { (Jayasena \& Dissanayake, } \\
2020 \text { ) }\end{array}$ \\
\hline
\end{tabular}

A hexane extract of polyherbal preparation consisting of seeds of Piper nigrum L., leaves of Murraya koenigii L. Sprengel, cloves of Allium sativum L. and the dried fruit rinds of Garcinia quaesita Pierre had demonstrated hypoglycaemic as well as hypolipidemic activities in vivo in streptozotocin-induced diabetic rats (Sampath et al., 2021).

Table 4 lists herbal preparations such as powders, ointments, decoctions, pills, and water extracts used against inflammatory conditions.

\section{Table 4}

Sri Lankan Herbal Preparations against Inflammatory Conditions

\begin{tabular}{|l|l|l|}
\hline Herbal preparation & Study stage & Findings \\
\hline $\begin{array}{l}\text { 1. "Sudarshana } \text { Powder" } \\
\text { consisting of 53 herbal } \\
\text { ingredients }\end{array}$ & $\begin{array}{l}\text { Pre-clinical } \\
\text { trial using } \\
\text { adjuvant } \\
\text { induced } \\
\text { arthritic rats. }\end{array}$ & $\begin{array}{l}\text { Significant reduction of foot pad } \\
\text { and ankle joint thicknesses and } \\
\text { white blood cell counts. Reduced } \\
\text { erythema and oedema in the ankle } \\
\text { joints and the foot pads. Normalized } \\
\text { body weight. } \\
\text { (Weerakoon et al., 2016) }\end{array}$ \\
\hline
\end{tabular}




\begin{tabular}{|l|l|l|}
\hline Herbal preparation & Study stage & Findings \\
\hline $\begin{array}{l}\text { 2. Ointment produced } \\
\text { using Curcuma albiflora } \\
\text { (Turmeric) }\end{array}$ & Clinical trial & $\begin{array}{l}\text { User satisfaction on disease } \\
\text { symptoms were 31 times greater } \\
\text { than the placebo. (Herath et al., } \\
\text { 2018) }\end{array}$ \\
\hline 3. Rasna Sapthakaya decoction & Clinical trial & $\begin{array}{l}\text { Significant reduction of erythrocyte } \\
\text { sedimentation rate and significant } \\
\text { increases of Haemoglobin, packed } \\
\text { cell volume and red blood cell } \\
\text { count. (Perera, 2018) }\end{array}$ \\
\hline $\begin{array}{l}\text { 4. Traditional medicine pill } \\
\text { consisting of Aloe vera, } \\
\text { Centella asiatica (Gotukola) } \\
\text { and Strychnos potatorum } \\
\text { (Clearing nut) }\end{array}$ & $\begin{array}{l}\text { In vitro test } \\
\text { albumin egg }\end{array}$ & $\begin{array}{l}\text { Significant anti-inflammatory effects } \\
\text { compared to the reference drug. } \\
\text { (Sarvaka et al., 2016) }\end{array}$ \\
\hline $\begin{array}{l}\text { 5. Aquous root extract of } \\
\begin{array}{l}\text { Syzygium Caryophyllatum } \\
\text { (South Indian plum) }\end{array}\end{array}$ & $\begin{array}{l}\text { In vitro test } \\
\text { using egg } \\
\text { albumin }\end{array}$ & $\begin{array}{l}\text { Significant anti-inflammatory } \\
\text { activity. (Heendeniya et al., 2018) }\end{array}$ \\
\hline
\end{tabular}

Other than the aforementioned research on anti-inflammatory effects of traditional herbal medicine, a study reported a total of 43 herbal plants used in anti-inflammatory preparations in which Coriandrum sativum was reported by most of the participants recruited within the Gampaha district (Napagoda et al., 2018).

Herbal preparations used against different conditions such as sun burns, urolithiasis, chronic sinusitis, and fertility and urinary issues with study stage and findings are presented in Table 5.

\section{Table 5}

Sri Lankan Herbal Preparations against Miscellaneous Disorders

\begin{tabular}{|c|c|c|c|}
\hline Herbal preparation & Disorder & Study stage & Findings \\
\hline $\begin{array}{l}\text { 1. Herbal paste consisting } \\
\text { of Brassica campestris } \\
\text { (Field mustard), Acorus } \\
\text { Calamus (Sweet flag), } \\
\text { Symplocos racemose } \\
\text { (Lodra), Rock Salt }\end{array}$ & $\begin{array}{l}\text { Sun burnt } \\
\text { skin }\end{array}$ & Clinical trial & $\begin{array}{l}\text { A significant increase in } \\
\text { skin fairness was reported } \\
\text { after treatment. Of the } \\
\text { total participants } 78 \% \\
\text { showed increse in skin } \\
\text { fairness level } \\
\text { (Abegunasekara \& } \\
\text { Wijesinghe, 2020) }\end{array}$ \\
\hline
\end{tabular}




\begin{tabular}{|c|c|c|c|}
\hline Herbal preparation & Disorder & Study stage & Findings \\
\hline $\begin{array}{l}\text { 2. Methanol extract of roots } \\
\text { of Asparagus falcatus } \\
\text { (Sicklethorn) }\end{array}$ & Urolithiasis & In vitro & $\begin{array}{l}\text { Prevention of crystal } \\
\text { nucleation by } 13.26 \% \text {; } \\
\text { aggregation by } 36.5 \% \text {, } \\
\text { dissolved weight of the } \\
\text { calculi by } 5.19 \% \text {. These } \\
\text { results are similar to } \\
\text { those demonstrated by the } \\
\text { positive control. } \\
\text { (Napagoda et al., 2019) }\end{array}$ \\
\hline $\begin{array}{l}\text { 3. Pitawakka nawaya } \\
\text { decoction consisting } \\
\text { of Phyllanthus amarus } \\
\text { (Gale of Wind), } \\
\text { Terminalia chebula } \\
\text { (Chebulic myrobalan), } \\
\text { Pygmaeopremna } \\
\text { herbacea (Bharangi), } \\
\text { Pipper chavya (Chavya), } \\
\text { Solanum trilobatum } \\
\text { (Purple fruited egg } \\
\text { plant), Tinospora } \\
\text { Cordifolia (heart-leaved } \\
\text { moonseed), Zingiber } \\
\text { officinalae (Ginger), } \\
\text { Pipper Nigrum (Black } \\
\text { pepper), and Piper } \\
\text { longum (Long pepper) }\end{array}$ & $\begin{array}{l}\text { Chronic } \\
\text { sinusitis }\end{array}$ & Clinical trial & $\begin{array}{l}\text { Reduction of heaviness } \\
\text { of the head in } 6.3 \% \text {; } \\
\text { headache in } \\
11.8 \% \text {; mild headache in } \\
\text { in } 7.7 \% \text {; itching inside } \\
\text { ear in } 14.3 \% \text {; nasal } \\
\text { obstruction in } 6.7 \% \text {; nasal } \\
\text { discharge in } 5.9 \% \\
\text { and abnormalities of } \\
\text { smell in } 66.7 \% \text {. } \\
\text { (Maragalawaththa et al., } \\
\text { 2010). }\end{array}$ \\
\hline $\begin{array}{l}\text { 4. Pala Kalyana Ghrita } \\
\text { poly herbal formula }\end{array}$ & $\begin{array}{l}\text { Fertility } \\
\text { Issues }\end{array}$ & $\begin{array}{l}\text { Pre-clinical } \\
\text { study using } \\
\text { Sprague } \\
\text { Dawley rats }\end{array}$ & $\begin{array}{l}\text { Significant increase in } \\
\text { testicular weight and } \\
\text { serum testosterone levels. } \\
\text { (Perera et al., 2018) }\end{array}$ \\
\hline $\begin{array}{l}\text { 5. Cold water extract of } \\
\text { Spilanthes acmella fresh } \\
\text { flowers }\end{array}$ & $\begin{array}{l}\text { Urinary } \\
\text { issues }\end{array}$ & $\begin{array}{l}\text { Pre-clinical } \\
\text { study using } \\
\text { albino rats }\end{array}$ & $\begin{array}{l}\text { Significant diuretic } \\
\text { activity similar to } \\
\text { furosemide. (Ratnasooriya } \\
\text { et al., 2004) }\end{array}$ \\
\hline
\end{tabular}

\section{The Way Forward}

Synergistic activity among phytochemicals seems to play a major role in the therapeutic properties of traditional herbal preparations. As such, expensive and timeconsuming experiments to identify a single bioactive phyto compound for a specific 
disorder or disease may be unnecessary, if it is feasible to select a compendium of phytocompounds with effective therapeutic bioactivity in drug development. Collaborative multidisciplinary research in the field of drug discovery using novel screening platforms (e.g., use of stem cell lines) and traditional medicine systems may generate fruitful outcomes in the identification and production of therapeutics. For example, traditional medicine practitioners and institutes can maintain open access data bases for herbs used in TM, together with their properties, which can be readily accessed by researchers for drug development. ${ }^{1}$ TM practitioners, in turn, can collaborate with modern medical institutes in using novel technology such as artificial intelligence to model disease-oriented drug targets for complex TM preparations. Exploring hitherto undisclosed areas in Sri Lankan Indigenous Medicine for critical diseases such as pneumonia associated with viral diseases such as SARS, MERS and Covid-19 would be another angle worth investigating.

As noted in the introduction, methods for the conservation and sustainable use of medicinal plants have already been established in Sri Lanka. However, understanding how to maintain this process is important. Figure 1 summarizes the factors and outcomes of the sustainable use of medicinal plants.

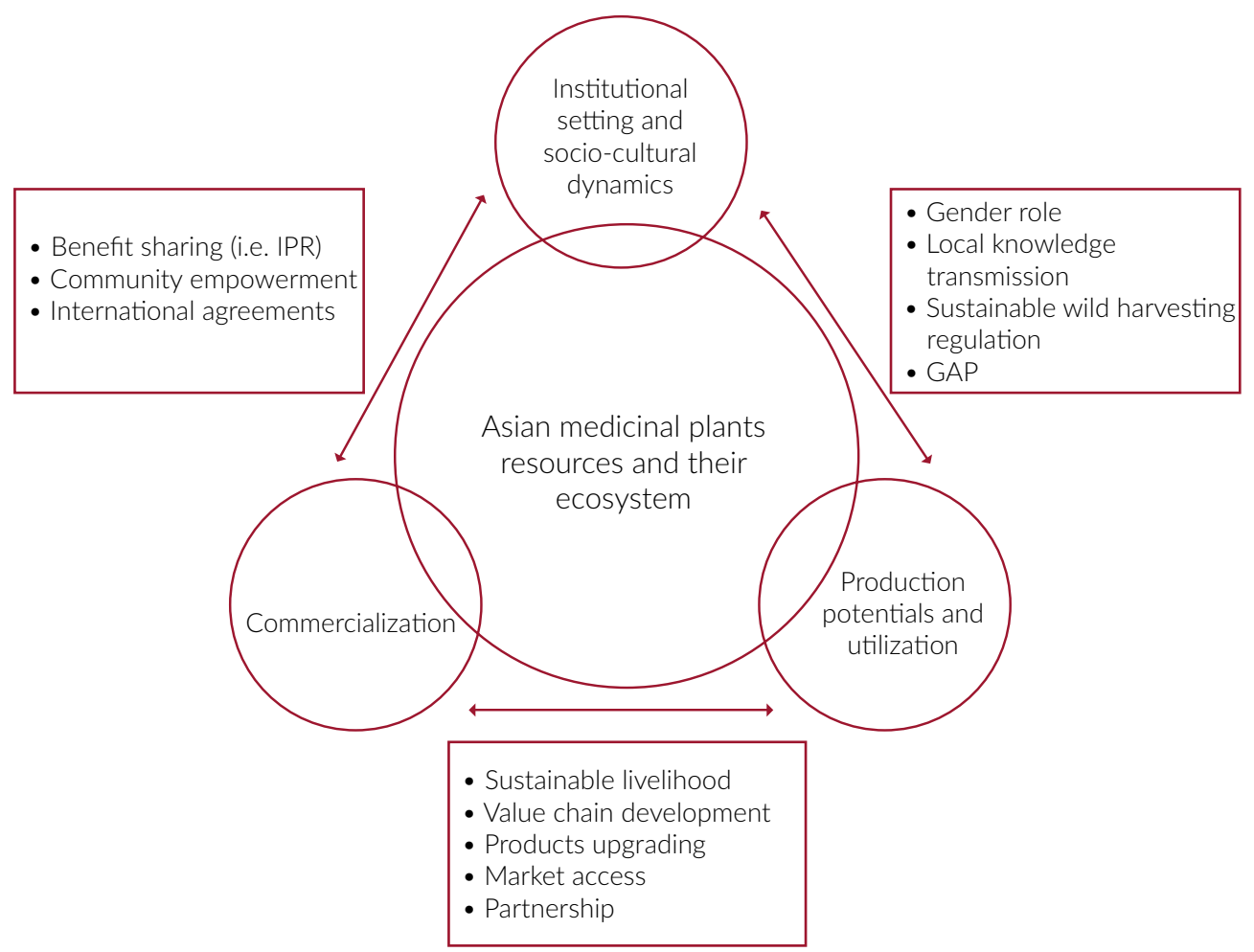

Figure 1

Schematic Presentation on Sustainable Management of Medicinal Plants

(Image Source: Astutik et al., 2019)

Note: Extracted from Astutik, S., Pretzsch, J., \& Ndzifon Kimengsi, J. (2019). Asian medicinal plants' production and utilization potentials: A review. Sustainability, 11(19), 5483. 
Therefore, it is important to carry out in-depth evidence based translational research on traditional medicine preparations, according to international standards, which may pave the path for appropriate dissemination of knowledge in the field of drug discovery.

As only $3.3 \%$ of medicinal plants are commercially cultivated globally, with the rest collected from the wild, Sri Lanka would benefit if medicinal plant cultivation elicited more government support (Astutik et al., 2019). As important is the recognition of the novel initiatives by private sector herbal product manufacturers to conserve and promote the sustainable usage of medicinal plants. Guidance by state institutes such as the Institute for Indigenous Medicine of the University of Colombo, the Gampaha Wickramaarachchi University of Indigenous Medicine, and other alternative medicine institutes would be a boon to the industry in its conservation management efforts. To avoid over exploitation of medicinal plants and prevent repetitive research, updated websites listing the medicinal plants of Sri Lanka with current research outcomes such as reported bioactivities, scientific validations, and clinical trials should be maintained. Data collected by state universities from research related to medicinal plant conservation should be used by the government to take these projects to the next level of implementation. Using inexpensive, human cellular platforms such as human stem cells isolated from biological waste (postpartum tissue and surgical waste) to screen traditional preparations and medicinal plants for their claimed bioactivities such as bone, cartilage, muscle, liver protection and regeneration would mark a future, hybridized, novel state of the art technology (Udalamaththa et al., 2016). Anti-cancer traditional preparations could be validated and commercialized with the use of cancer stem cell platforms within a shorter time-period. As genome sequencing methods are well established in modern medicine, personalized medicine treatment could be promoted by investigating the effectiveness of herbal preparations on diseases caused by multifactorial genetic aetiologies.

State support for such activities should be fostered by the State Ministry of Indigenous Medicine under the purview of the Ministry of Health, Sri Lanka. Such a strategy would enable local scientists and local drug companies to compete with global pharmaceutical giants, which could be advantageous to the Sri Lankan economy. Figure 2 demonstrates this with cues from TM, the time period for the development of a drug may surmount by at least 10 years. 


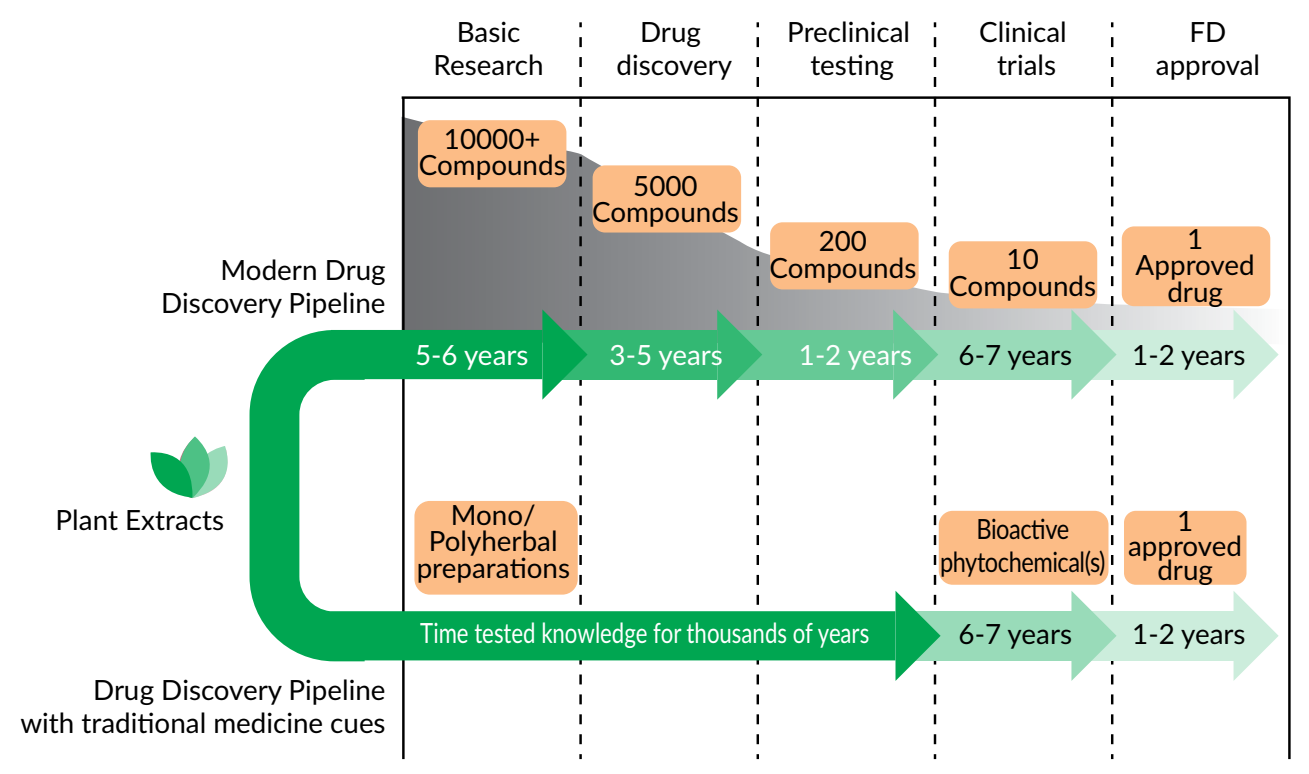

Figure 2

Comparison of Drug Discovery Time Lines Based on Modern Technology and with Cues from Traditional Medicine

\section{Conclusions}

This article discussed time tested herbal remedies and whole plant extracts used in Sri Lankan Traditional Medicine for the treatment of a wide range of diseases/disorders. When tested via modern scientific platforms or clinical studies these may lead to a considerably shorter turnover time and less expenditure in the development of modern drugs. The article also stressed that these remedies may be more effective and non-toxic because whole or crude preparations, with several phytocompounds acting synergistically on multiple molecular targets, are better than an isolated single compound which is the goal of current drug development. The use of novel technology to screen for important bioactivity, reporting scientific findings, proceeding with preclinical and clinical studies, and drug development with several compounds or whole preparations would be advantages to countries such as Sri Lanka, where invaluable and effective traditional medicine solutions for a wide range of diseases have been in use. If accepted by the global market, a socioeconomically sustainable future could be at hand for Sri Lankan cultivators of medicinal plants and local pharmaceutical industrialist, as there would be a continuous demand for quality, fresh medicinal plant material for the production lines. This would also create an uninterrupted flow of foreign exchange into the country.

1. National monographs of herbs used in Sri Lanka and Pharmacopeia are currently available (Astutik et al., 2019). 


\section{References}

Abegunasekara, N. S., \& Wijesinghe, K. D. A. (2020). Evaluation of the effect and efficacy of herbal application mentioned in Sharangadhara Samhita in enhancing the skin complexion. International Journal of Ayurveda, 5(6), 1-14.

Astutik, S., Pretzsch, J., \& Ndzifon Kimengsi, J. (2019). Asian medicinal plants' production and utilization potentials: A review. Sustainability, 11(19), 5483. https://doi.org/10.3390/su11195483

Caesar, L. K., \& Cech, N. B. (2019). Synergy and antagonism in natural product extracts: when 1+ 1 does not equal 2. Natural Product Reports, 36(6), 869-888. https://doi.org/10.1039/C9NP00011A

Daily FT. (2021, April 7). Nature's Secrets champions greener, healthier Sri Lanka. https:// www.ft.lk/sustainability_environment/Nature-s-Secrets-champions-greener-healthier-SriLanka/10519-715978

Dias, D. K., Atukorala, C., Amaratunga, N. D. S., Perera, B., \& Karunathilake, L. P. A. (2018). The effects of Emblica officinalis and Tinospora cordifolia herbal treatment on the prognosis of squamous cell carcinoma of the buccal mucosa and tongue. Journal of Oral and Maxillofacial Surgery, Medicine, and Pathology, 30(1), 21-29. https://doi.org/10.1016/j.ajoms.2017.08.009

Dissanayaka, D. M. L. C., Nilakarawasam, N., Somaratne, S., Weerakoon, S. R., \& Ranasinghe, C. (2019). Effect of Phyllanthus maderaspatensis L. crude methanolic extract on diet induced hypercholesterolemia in Wistar albino rats (Mus norvegicus albinus). Ceylon Journal of Science, 48(3), 285-291. http://doi.org/10.4038/cjs.v48i3.7653

Dissanayake, D. M. R. K., Wijayabandara, M. D. J., \& Ratnasooriya, W. D. (2016). Hypoglycaemic and antihyperglycaemic activities of an aqueous leaf extract of Adenanthera pavonina (fabaceae) in rats. Int J Pharm Res \& Allied Sci, 5(1), 34-8.

Edwards, S., Da-Costa-Rocha, I., Lawrence, M. J., Cable, C., \& Heinrich, M. (2012). Use and efficacy of herbal medicines: Part 1-Historical and traditional use. The Pharmaceutical Journal, 289(7717), 161-162.

Fleming, N. (2018). Computer-calculated compounds. Nature, 557(7707), S55-S57.

Ganewatte Medicinal Plant Gardens. (2009). Government Information Centre. Retrieved October 19, 2021, from https://www.gic.gov.lk/gic/index.php/en/component/info/?id=1455\&task=info

Hapuarachchi, S. D., Suresh, T. S., \& Senerath, W. T. P. S. K. (2011). A preliminary study of oral hypoglycemic activity of the ethanol and water extract of Munronia pinnata in Healthy Wistar rats. Sri Lanka Journal of Indigenous Medicine, 1, 1-4.

Harshamali, K.A.D.T., \& Sakunthala, H.S. (2019). Evaluation of the effect and efficacy of herbal powder preparation derived from 'Thalpathe Piliyam' in the management of type II Diabetes mellitus [paper presentation], Young Ayurveda Researchers' and Innovators' Symposium, Gampaha Wickramarachchi Ayurveda Institute, University of Kelaniya, Yakkala, Sri Lanka.

Heendeniya, S., Ratnasooriya, W. D., \& Pathirana, R. N. (2018). In vitro investigation of antiinflammatory activity and evaluation of phytochemical profile of Syzygium caryophyllatum. Journal of Pharmacognosy and Phytochemistry, 7(1), 1759-1763. 
Herath, I. C., Wijayasiriwardene, T. D. C. M. K., \& Premakumara, G. A. S. (2018). Study on effectiveness and user satisfaction of anti-inflammatory ointment of Curcuma albiflora Thw. in Bentota divisional secretariat, Southern Sri Lanka. Ruhuna Journal of Science, 9(2).

Jalpa, H. J. \& Pandya, K. (2017). Arka Kalpana - A Review W.S.R. To Distillation. International Journal of Advanced Research (IJAR), 5(7), 867-872.

Jayasena, R. M. D., \& Dissanayake, K. G. C. (2020). A Clinical Study on the Effect and Efficacy of Traditional Formulation Derived by Ola Leaves Manuscript in the Management of Overweight and Obesity. International Journal of Scientific and Research Publications, 10(1), 709-711. http://dx.doi.org/10.29322/IJSRP.10.01.2020.p97103

Jia, W., Gao, W. Y., Yan, Y. Q., Wang, J., Xu, Z. H., Zheng, W. J., \& Xiao, P. G. (2004). The rediscovery of ancient Chinese herbal formulas. Phytotherapy Research: An International Journal Devoted to Pharmacological and Toxicological Evaluation of Natural Product Derivatives, 18(8), 681-686. https://doi.org/10.1002/ptr.1506

Kannangara, A. P. (2016). The history of dermatology, venereology, and dermatopathology in different countries-Sri Lanka. Global Dermatology, 2(7). https://doi.org/10.15761/GOD.1000S013

Kennedy, D. O., \& Wightman, E. L. (2011). Herbal extracts and phytochemicals: plant secondary metabolites and the enhancement of human brain function. Advances in Nutrition (Bethesda, Md.), 2(1), 32-50. https://doi.org/10.3945/an.110.000117

Keshala, K. K., Bandara, A. M. P. W., Padumadasa, C., \& Peiris, L. D. C. (2021). Bioactivities and GC-MS profiling of Malewana Madhumeha Choorna polyherbal hot infusion. South African Journal of Botany, 140, 194-203. https://doi.org/10.1016/j.sajb.2021.04.015

Kumar, M., Rawat, S., Nagar, B., Kumar, A., Pala, N. A., Bhat, J. A., ... \& Kunwar, R. (2021). Implementation of the use of ethnomedicinal plants for curing diseases in the Indian Himalayas and its role in sustainability of livelihoods and socioeconomic development. International Journal of Environmental Research and Public Health, 18(4), 1509. https://doi.org/10.3390/ ijerph18041509

Lindamulage, I. K., \& Soysa, P. (2016). Evaluation of anticancer properties of a decoction containing Adenanthera pavonina L. and Thespesia populnea L. BMC Complementary and Alternative Medicine, 16(1), 1-8. https://doi.org/10.1186/s12906-016-1053-9

Liyanagamage, D. S. N. K., Jayasinghe, S., Attanayake, A. P., \& Karunaratne, V. (2021). Dual mechanisms of a Sri Lankan traditional polyherbal mixture in the improvement of pancreatic beta cell functions and restoration of lipoprotein alterations in streptozotocin induced diabetic rats. Journal of Ethnopharmacology, 267, 113613. https://doi.org/10.1016/j.jep.2020.113613

Mahindapala, R. (2004). Medicinal plants: conservation and sustainable use in Sri Lanka. IK Notes, World Bank.

Maragalawaththa, M. K., Ediriweera, E. S., \& Chandimarathne, P. B. (2010). A clinical trial of Sri Lankan traditional decoction of Pitawakka Navaya in treatment of Kaphaja Shirsha Shoola (chronic sinusitis). Ayu, 31(2), 193. https://doi.org/10.4103/0974-8520.72390 
Mendis, A. S., Thabrew, I., Samarakoon, S. R., \& Tennekoon, K. H. (2015). Modulation of expression of heat shock proteins and apoptosis by Flueggea leucopyrus (Willd) decoction in three breast cancer phenotypes. BMC Complementary and Alternative Medicine, 15(1), 1-14. https://doi. org/10.1186/s12906-015-0927-6

Mukherjee, P. K., Banerjee, S., \& Kar, A. (2018). Exploring synergy in ayurveda and traditional Indian systems of medicine. Synergy, 7, 30-33. https://doi.org/10.1016/j.synres.2018.10.003

Nagenahiru Foundation. (2010). Conservation of Medicinal Plants.

Napagoda, M. T., Sundarapperuma, T., Fonseka, D., Amarasiri, S., \& Gunaratna, P. (2018). An ethnobotanical study of the medicinal plants used as anti-inflammatory remedies in Gampaha District, Western Province, Sri Lanka. Scientifica, 2018. https://doi.org/10.1155/2018/9395052

Napagoda, M., Madhumadhavi, N., Vimukthi, K., Malkanthi, S., \& Abayawardana, K. (2019). In vitro Antiurolithiatic Potential in Asparagus falcatus L., A Folklore Medicinal Plant in Sri Lanka. Journal of PharmaSciTech. 9: 28-31. https://doi.org/10.33981/JPST.2020.v09i01.005

Pan, L., de Blanco, E. J. C., \& Kinghorn, A. D. (2009). Plant-derived natural products as leads for drug discovery. Plant-derived natural products (pp. 547-567). Springer. https://doi. org/10.1007/978-0-387-85498-4_24

Pan, S. Y., Litscher, G., Gao, S. H., Zhou, S. F., Yu, Z. L., Chen, H. Q., ... \& Ko, K. M. (2014). Historical perspective of traditional indigenous medical practices: the current renaissance and conservation of herbal resources. Evidence-Based Complementary and Alternative Medicine, 2014. https://doi.org/10.1155/2014/525340

Pallie, M., Perera, P. K., Goonasekara, C. L., Kumarasinghe, N., \& Arawwawala, M. (2020). Efficacy and safety of freeze-dried form of Tragia involucrata L. decoction in treating diabetes: a randomized controlled clinical trial. Clinical Trials in Degenerative Diseases, 5(3), 31-36.

Pathiranage, V. C., Thabrew, I., Samarakoon, S. R., Tennekoon, K. H., Rajagopalan, U., \& Ediriweera, M. K. (2020). Evaluation of anticancer effects of a pharmaceutically viable extract of a traditional polyherbal mixture against non-small-cell lung cancer cells. Journal of Integrative Medicine, 18(3), 242-252. https://doi.org/10.1016/j.joim.2020.02.007

Perera, K. C., Wijeratne, S., Lokuhetty, D., \& Mathew, D. (2018). Sperm parameters and testosterone levels of Sprague Dawley rats with Pala Kalyana Ghrita: a traditional formula. European Journal of Biomedical, 5(5), 104-108.

Perera, P. K. (2018). Ayurveda and Sri Lankan traditional medicine approaches in joint disorders (Sandhigata Roga) and clinical trials. Proceedings of 20th International Ayurveda Symposium, Germany.

Perera, P. R. D., Ekanayake, S., \& Ranaweera, K. K. D. S. (2014). Antiglycation and antioxidant activities of a ready to serve herbal drink of Syzygium cumini bark extract. Medicinal \& Aromatic Plants, 3, article 148. http://dx.doi.org/10.4172/2167-0412.1000148 
Pushpamali, W. A. I., \& Chandrasiri, W. A. L. (2016). Evaluation of the effect and efficacy of cold water extract of new herbal formulation on serum lipid levels in the management of hyperlipidaemia [paper presentation]. Proceedings in Medical, Allied Health, Basic and Applied Sciences, 9th International Research Conference - KDU, Sri Lanka, 38-42

Rajagopalan, U., Samarakoon, S. R., Tennekoon, K. H., Malavige, N., \& De Silva, E. D. (2018). Screening of five Sri Lankan endemic plants for anti-cancer effects on breast cancer stem cells isolated from MCF-7 and MDA-MB-231 cell lines. Tropical Journal of Pharmaceutical Research, 17(9), 1825-1832. https://doi.org/10.4314/tjpr.v17i9.21

Rangika, B. S., Dayananda, P. D., \& Peiris, D. C. (2015). Hypoglycemic and hypolipidemic activities of aqueous extract of flowers from Nycantus arbor-tristis L. in male mice. BMC Complementary and Alternative Medicine, 15(1), 1-9. https://doi.org/10.1186/s12906-015-0807-0

Ratnasooriya, W. D., Pieris, K. P. P., Samaratunga, U., \& Jayakody, J. R. A. C. (2004). Diuretic activity of Spilanthes acmella flowers in rats. Journal of Ethnopharmacology, 91(2-3), 317-320. https://doi.org/10.1016/j.jep.2004.01.006

Ratnayake, R. S. S., \& Kariyawasam, C. S. (2007). Conservation and use of wild-harvested medicinal plants in Sri Lanka. Crop wild relative conservation and use. 625-631.

Samanmali, B. L. C., Gunatilake, M., Guneratne, R. D., \& Perera, T. R. K. (2014). Hypoglycaemic effect of the methanolic extract of Aporusa lindleyana leaves on rats. Journal of the National Science Foundation of Sri Lanka, 42(2). http://dx.doi.org/10.4038/jnsfsr.v42i2.6995

Samarakoon, S. R., Kotigala, S. B., Gammana-Liyanage, I., Thabrew, I., Tennekoon, K. H., Siriwardana, A., \& Galhena, P. B. (2014). Cytotoxic and apoptotic effect of the decoction of the aerial parts of Flueggea leucopyrus on human endometrial carcinoma (AN3CA) cells. Tropical Journal of Pharmaceutical Research, 13(6), 873-880. https://doi.org/10.4314/tjpr.v13i6.7

Samarakoon, S. R., Thabrew, I., Galhena, P. B., De Silva, D., \& Tennekoon, K. H. (2010). A comparison of the cytotoxic potential of standardized aqueous and ethanolic extracts of a polyherbal mixture comprised of Nigella sativa (seeds), Hemidesmus indicus (roots) and Smilax glabra (rhizome). Pharmacognosy Research, 2(6), 335. https://dx.doi.org/10.4103/0974-8490.75451

Sampath, S. N. T. I., Jayasinghe, S., Attanayake, A. P., \& Karunaratne, V. (2021). Sub-acute antihyperlipidemic and antihyperglycemic activity of the hexane extract of a polyherbal mixture in streptozotocin-induced diabetic rats. Ceylon Journal of Science, 50(3), 279-287.

Sarvaka, S., Galgamuwa, G. L. S., Siriwardene, U., Silva, A. R. N., \& Kumarasinghe, N. (2016). Testing the Anti-inflammatory Activity of Sri Lankan traditional medicine pill using albumin denaturation method [paper presentation]. Proceedings in Medical, Allied Health, Basic and Applied Sciences, 9th International Research Conference - KDU, Sri Lanka, 12-15.

Shahrajabian, M. H., Sun, W., \& Cheng, Q. (2019). Traditional Chinese medicine and agriculture; organic life and sustainability for future. GSC Biological and Pharmaceutical sciences, 7(1). https://doi.org/10.30574/gscbps.2019.7.1.0052 
Sharma, C. P. (2000). Ravana: A great scholar and scientist. Journal of Gampaha Wickramarachchi Ayurveda Institute, 2(1), 19-20.

Sudasinghe, H. P., \& Peiris, D. C. (2018). Hypoglycemic and hypolipidemic activity of aqueous leaf extract of Passiflora suberosa L. PeerJ, 6, e4389. https://doi.org/10.7717/peerj.4389

Tilburt, J. C., \& Kaptchuk, T. J. (2008). Herbal medicine research and global health: an ethical analysis. Bulletin of the World Health Organization, 86, 594-599. https://dx.doi. org/10.2471\%2FBLT.07.042820

Udalamaththa, V. L., Jayasinghe, C. D., \& Udagama, P. V. (2016). Potential role of herbal remedies in stem cell therapy: proliferation and differentiation of human mesenchymal stromal cells. Stem Cell Research \& Therapy, 7(1), 110-117. https://doi.org/10.1186/s13287-016-0366-4

Vambe, M., Aremu, A. O., Chukwujekwu, J. C., Finnie, J. F., \& Van Staden, J. (2018). Antibacterial screening, synergy studies and phenolic content of seven South African medicinal plants against drug-sensitive and-resistant microbial strains. South African Journal of Botany, 114, 250-259. https://doi.org/10.1016/j.sajb.2017.11.011

Wageesha, N. D. A., Soysa, P., Atthanayake, K., Choudhary, M. I., \& Ekanayake, M. (2017a). A traditional poly herbal medicine "Le Pana Guliya" induces apoptosis in HepG 2 and HeLa cells but not in CC1 cells: an in vitro assessment. Chemistry Central Journal, 11(1), 1-2. https://doi. org/10.1186/s13065-016-0234-4

Wageesha, N. D. A., Soysa, P., Atthanayake, K., Choudhary, M. I., \& Ekanayake, M. (2017b). Evaluation of antioxidant and polyphenolic content of a Sri Lankan poly herbal formulae and assessment of its in vitro antiproliferative activity on RD and MCF-7 cancer cells compared to healthy CC1 cells. Cancer Study and Therapeutics, 2(3), 1-8.

Weerakoon, W. A. S. S., Perera, P. K., Gunasekara, D., \& Suresh, T. S. (2016). Chronic antiinflammatory activity of Sudarshana powder on adjuvant-induced arthritis in rats [paper presentation]. Proceedings of the Peradeniya University International Research Sessions, Sri Lanka, 205.

World Health Organization. (2000). General guidelines for methodologies on research and evaluation of traditional medicine (No. WHO/EDM/TRM/2000.1).

Zhou, X., Seto, S. W., Chang, D., Kiat, H., Razmovski-Naumovski, V., Chan, K., \& Bensoussan, A. (2016). Synergistic effects of Chinese herbal medicine: a comprehensive review of methodology and current research. Frontiers in Pharmacology, 7, 20 